\title{
TECHNIKPHOBIE UND SEXISMUS. EINE INTERVIEWSTUDIE ZU ERFAHRUNGEN WEIBLICHER DJS IN FRANKFURT AM MAIN
}

\section{Corinna Jean Zimmermann}

Im Hinblick auf die DJ-Szene mag man zunächst nicht an stereotype Rollenbilder denken, da diese junge Kultur ein ausgeglichenes Geschlechterverhältnis propagiert. Bei eingehender Analyse kann man jedoch ein geschlechtliches Ungleichgewicht unter den agierenden DJs ausmachen, wobei sich dabei die Frage stellt, ob dieser klischeehafte Eindruck der Realität entspricht und - wenn ja - worin dieses Phänomen begründet ist. Als adäquate Methode zur Erörterung dieser Fragen bot sich eine qualitative Interviewstudie an, die den Erfahrungen und Einschätzungen weiblicher DJs in der Technoszene im Kontext eines Szeneumfelds nachging. Dies ist besonders im Hinblick auf das Selbstverständnis der Technoszene interessant, die sich als offene Szene versteht - als eine Szene, in der jeder sein darf, wie »er« will, und Männer und Frauen gleichgestellt sind. Aber schon im Standardwerk über Techno von Anz und Walder wird eine Schieflage festgestellt:

»[E]ine Frage bleibt: Warum schreiben fast nur Männer über Techno - über männliche DJs? Wir hatten uns das anders vorgestellt: In einer jungen und lebendigen Kultur, in der die Plätze noch nicht verteilt sind, an deren Partys die Frauen sich freier und selbstbestimmter bewegen können als anderswo« (Anz/Walder 1995: 7).

Hier kommen mehrere Aspekte zum Ausdruck: Erstens ist von einem geschlechtlichen Ungleichgewicht bei wichtigen Protagonisten der Szene die Rede, den DJs und den Journalisten, das zweitens mit dem Selbstverständnis der Szene - konnotiert mit »offener « Kultur und Distanzierung von traditionellen Geschlechterrollen - kollidiert. Drittens stellt man diesen Umstand schon 1995 fest, also bereits in der Anfangszeit des Techno. Dies führte zu folgenden Fragen: Sind die Verhältnisse, fast 15 Jahre später, immer noch ähnlich wie damals? Was sind die Gründe dafür? Wie gehen die Frauen damit um? Welche Erfahrungen machen sie? 
Um diese Fragen zu erörtern, wurden sechs weibliche DJs interviewt, die in Frankfurt und Umgebung auflegen. Hierfür wurde ein qualitativer Leitfragebogen erarbeitet, der sich an vorausgehenden Interviewstudien und Berichten orientiert (vgl. Stauder 2004). Die ca. einstündigen Interviews wurden transkribiert und qualitativ nach Themenschwerpunkten ausgewertet. ${ }^{1}$

\section{Geschlechterverhältnisse im Techno}

Barbara Stauber führte 2004 eine Studie zur Rollenverteilung in Jugendkulturen durch und widmete sich u.a. auch der Technokultur; dabei trat zutage, dass »die Männer sich tendenziell eher um die Technik oder um die Logistik kümmern, die Frauen tendenziell eher um Getränke und Dekoration, Kasse machen beide« (Stauber 2004: 92). Hier kommt zum Ausdruck, dass Frauen zwar in der Szene vorhanden sind, aber nicht unbedingt als Hauptakteure. Dies spiegelt den Eindruck eines traditionellen, konservativen Rollendenkens, das sich sogar in eine Art Geschlechterhierarchie ausbildet, was dem Selbstbild der Szene widerspricht. Aber worin liegt dieses Selbstbild begründet? Die Offenheit der Techno-Szene wird in erster Linie mit freiem Körperkult assoziiert. In der Techno-Szene können Frauen frei verkehren, da das typische »Balzverhalten« keine Rolle spielt (vgl. Coers 2000: 90-94). Das Tanzen steht im Vordergrund und es gilt das Motto »Bewegung statt Sex« (Gardizi 2006: 195). Dabei sollte aber zwischen Mainstream und Underground unterschieden werden. Bei der Love Parade und in großen Clubs scheint ein anderes Ideal zu herrschen als im kleinen Undergroundclub. Dort dominiert das Ideal des perfekten Körpers muskelbepackter Männer und schlanker, attraktiver Frauen, die gerne Haut zeigen. Dies widerspricht freilich dem ursprünglichen Leitgedanken der TechnoKultur, der Toleranz gegenüber Allem und Jedem abseits eines allgemein anerkannten Schönheitsideals. Dass dieses Ideal unter den DJs nicht anzutreffen ist, wird in Gardizis Aufsatz beschrieben. Er zitiert Hans Nieswandt, seines Zeichens DJ und Produzent:

»Für die Minderheit weiblicher DJs stellt sich immer das gleiche Dilemma. Jeder Auftritt gerät potentiell und unvermeidlich zu einem Statement weiblicher Attitüden im Spannungsfeld zwischen Riot-Grrrl-Power und DiscoLuder. Der Prüfstein heißt nicht: Wie legt sie auf? Sondern: Wie legt sie auf, so als Frau?«(Gardizi 2006: 194).

1 Die Auswertung erfolgte als strukturierende Inhaltsanalyse nach Mayring 2000: 473. 
Eine Erklärung für die geringe Präsenz weiblicher DJs gibt Isabel Lorenz in ihrer Magisterarbeit (Lorenz 2007); sie argumentiert mit der »glass-ceiling «, einer unsichtbaren Grenze, an die Frauen auf dem Weg »nach oben« stoßen; quasi ein Endpunkt in der Karriere, über den Frauen nicht hinaus kommen, weil sie Frauen sind. ${ }^{2}$ Die Szene ist demnach dominiert von einem MännerNetzwerk, in das keine Frauen aufgenommen werden. In der Literatur wird dies kontrovers diskutiert, die Problematik ist aber erkannt (vgl. Braunersreuther/Maida 2000: 66; Skywalker 2000; Zimmermann 2008: 15f.). An solche geschlechtertypischen Phänomene anschließend, wird die Technobewegung auch als Bewegung mit feministischen Elementen ausgelegt. Ob die heutige Clubszene jedoch solche Elemente beinhaltet, wird von Gilbert kritisch gesehen:

»Coming from the early 1990s rave scene, I was initially suspicious of this development. The glamourisation of the dance-floor; the marketing of Ibiza as a holiday destination for those seeking casual sex rather than psychedelic togetherness; the popularity of dress-codes at over-priced ssuper-clubs; the complete disappearance of asexual clothing from women's clubbing wardrobes; the increasing sense that it was becoming impossible for women to go out dancing without being physically harassed by men who, never having experienced the asexual Acid House utopia, took their confident modes of self-expression (or just their presence of the dancefloor) as a come-on: all seemed to bespeak a general decline in the politically progressive character of UK dance culture. I still think that this is true across a large number of sites, and it is true that the idea of the post-rave dancefloor as a space at which a feminist project is enacted has largely disappeared « (Gilbert 2006: 188).

\section{Techno in Frankfurt}

Um die Rolle weiblicher DJs in der Frankfurter Szene zu analysieren, ist es sinnvoll, die Techno-Szene in Frankfurt im Allgemeinen und ihre Entwicklung näher zu betrachten. Als sich in den 1980er Jahren die so genannte Techno-Musik ausbreitete, gab es zwei bedeutende Zentren in Deutschland, Berlin und Frankfurt am Main. Die Diskothek Omen, die von Sven Väth, der als Wegbereiter des Techno gehandelt wird, Michael Münzing und Matthias Martinsohn gegründet wurde, galt bis 1999 »als das Herz der deutschen

2 Nach der Präsenz einer solchen »glass-ceiling « habe ich auch meine Interviewpartnerinnen gefragt. Hierzu gab es divergierende Meinungen. Es gebe wohl einen Punkt, an dem es nicht weiter gehe, aber dies sei nicht durch das Geschlecht begründet, so die befragten DJs (vgl. Zimmermann 2008: 50ff.). 
Techno-Szene « (Kemper 2004: 49). 1987 und in den folgenden Jahren hatten mehrere aus Frankfurt stammende DJs große Erfolge mit ihren Tracks. Ebenfalls 1987 wurde in Frankfurt die Zeitschrift Groove gegründet, die bis heute zu den wichtigsten Techno-Zeitschriften zählt; ihren Redaktionssitz hat sie seit 2001 jedoch in Berlin. Wichtige Orte der Frankfurter Technoszene sind heute Cocoon (wurde u.a. von Sven Väth 2004 eröffnet), Robert Johnson, U60311, Tanzhaus West und Vinylbar. ${ }^{3}$

Über die Jahre haben sich die Bedingungen in Frankfurt verändert, die Szene ist laut der Befragten einfacher zugänglich als vor ca. zehn Jahren. Eine DJ sagte dazu im Interview: »Es ist nicht mehr das Prinzip >König und sein Hofstaat<, heute ist es eher ein Nebeneinander « (F: 3). ${ }^{4}$ Trotzdem macht sich eine Hierarchie bemerkbar: An oberster Stelle steht das Cocoon, wo nur international bekannte DJs auftreten, dann darunter Clubs, in denen lokale und internationale DJs spielen, z.B. U60311 und Tanzhaus West, und dann Clubs, in denen nur lokale Größen spielen.

\section{Methode}

Um das Phänomen der Geschlechterverhältnisse im Techno zu untersuchen, wurde als exploratives Vorgehen eine qualitative Interviewstudie gewählt. Fünf bis zehn weibliche DJs sollten interviewt werden. Nach diesen habe ich in diversen Clubs gesucht, d.h. ich habe die Clubbetreiber angeschrieben und nach Kontaktdaten gefragt. Anfangs gab es neun Zusagen, jedoch kam mit drei DJs aus verschiedenen Gründen kein Interview zustande. Die Untersuchung wurde anhand von Leitfragen-Interviews durchgeführt, ein Fragebogen zu biografischen Daten wurde vorangestellt. Die Leitfragen lassen sich in folgende Unterpunkte gliedern:

- Geschlechterverhältnisse \& Gender

- Beruflicher Alltag

- Netzwerke \& Kolleginnen

- Motivation

- Image

- Standort Frankfurt

3 Diese Lokalitäten wurden von den von mir interviewten weiblichen DJs immer wieder genannt (vgl. Zimmermann 2008: 20ff.).

4 Die Aussagen der DJs wurden anonymisiert. Jede Befragte erhielt einen Buchstabencode $(A, B, C, D, E, F)$, anhand dessen die einzelnen Aussagen den jeweiligen DJs zugeordnet werden können. 
Von diesen Themenschwerpunkten erhielt die Geschlechterfrage die größte Gewichtung. Die Interviewaussagen sind im Folgenden nach der MontageTechnik zusammengestellt und thematisch geordnet, sodass Übereinkünfte, aber auch entgegen gesetzte Meinungen deutlich erkennbar werden.

\section{Die Interviewpartnerinnen}

Die sechs befragten DJs wohnen und spielen regelmäßig in Frankfurt und Umgebung. Sie treten unter den Namen Anne Anderson, Kia, Maya, Melane Cartier, Sophie Nixdorf und Vera auf und sind zwischen 23 und 43 Jahren alt. Sie werden ca. 30 Mal im Jahr gebucht, mit Ausnahme von Vera - sie ist international bekannt und tritt über 50 Mal im Jahr auf; die anderen DJs beschränken sich eher auf den Raum Frankfurt. Bis auf eine der Befragten können oder wollen sie ihren Lebensunterhalt nicht nur vom Auflegen und Produzieren bestreiten. Eine der Befragten lebt zwar hauptberuflich von der Musik, aber nicht nur als DJ, sondern auch als Veranstalterin, Medienpädagogin und Produzentin. Eine weitere übt zusätzlich einen Mini-Job aus, bestreitet aber zwei Drittel ihres Einkommens durch das Auflegen. Drei der DJs arbeiten hauptberuflich in einem anderen Beruf, als Krankenschwester, Werbekauffrau und Bildbearbeiterin. Die Gagen der Interviewpartnerinnen variieren je nach Bekanntheitsgrad zwischen 100 und über 1000 Euro pro Abend (Stand November 2008). Auf die Frage, ob sie Erfahrungen im Instrumentalspiel hätten, wurde sehr unterschiedlich geantwortet. Manche spielen mehrere Instrumente und hatten jahrelang klassischen Musikunterricht, manche spielen kein anderes Instrument außer dem Plattenspieler.

\section{Gründe für die geringe Präsenz weiblicher DJs}

Dass eine Ungleichheit der Geschlechter vorhanden ist, zeigt sich u.a. darin, dass ganz offen von einem »Männerbusiness « gesprochen wird:

»Ich spüre natürlich schon, dass meine Ansprechpartner alle Männer sind. Und in dem Punkt spüre ich natürlich, dass es ein Männerbusiness ist. Da stoße ich auf Grenzen. [...] Und das ist was, wo ich mich benachteiligt fühle. Ich will keine falsche Solidarität von einer Frau, das wäre Quatsch, aber trotzdem wäre es schön, mal mit Frauen zusammenzuarbeiten. Gerade im >höheren Bereich mal mit einer Frau was zu machen. Aber es gibt fast keine« (A: 9). 
Ein häufig genannter Aspekt ist, dass die Männer schon länger im Geschäft sind und auch aus diesen Gründen erfolgreicher sind. Die Frage, warum Männer länger im Geschäft sind, bleibt aber meist unbeantwortet. Diesen Umstand als Gender-Problematik zu betrachten, wurde nicht unbedingt angenommen. Dennoch wurden bestimmte geschlechtertypische Eigenschaften angeführt:

»Das könnte aber auch damit zusammenhängen, dass die Männer früher angefangen haben. Also diejenigen, die wirklich international richtig bekannt sind, viel Kohle verdienen und viel unterwegs sind, sind alles alte Hasen. Das sind Leute, die mindestens zwanzig Jahre im Geschäft sind, sich einen Namen gemacht haben und die Konkurrenz hinter sich gelassen haben. Es gibt Frauen, die nachziehen, z.B. Magda, Monika Kruse und Miss Kittin, aber die Pole-Positions sind schon noch von Männern besetzt. Aber ich denke, dass das daran liegt, dass die Frauen später angefangen haben als die Männer. [...] Vielleicht haben Frauen auch eher ein `Sicherheitsdenken tiger. Eventuell denken sie, >Ach, das ist kein gescheiter Beruf « und sagen sich, >Da gehe ich lieber den sicheren Weg und lerne lieber Bürokauffrau<. Das könnte es auch sein. Männer denken eher, >Ich schaff das‘, >Ich bin cool«« (F: 7).

Hier kommt zum Ausdruck, dass Männer anscheinend mehr Selbstvertrauen haben. Die niedrige Präsenz von Frauen hinter den Plattentellern wird auch mit der Sozialisation von Mädchen und Jungen und mit der Familienkonstellation begründet, bei der die Frauen den Beruf oftmals für die Familie aufgeben:

»Vielleicht weil Frauen nicht so dazu erzogen wurden, den Affen zu machen. So ein DJ-Dasein hat ja schon viel mit Entertainment, Rampensau und Leuteunterhalten, Nach-vorne-Gehen zu tun. Das ist schon so eine Erziehungssache. [...] Und wenn sie dann doch Kinder kriegen, geben sie eher ihre Hobbys auf als die Männer« (D: 7).

Das mangelnde Selbstvertrauen der Frauen macht sich aber auch bei der Gage bemerkbar, so wird erwähnt:

» Für eine Frau legst du gut auf< und >Cool, eine Frau mal hinter den Plattentellern zu sehen $<$. Was ja gut gemeint ist, aber doch negativ ist. Hinter den Sprüchen verbirgt sich die Aussage, >Cool, dass sich das eine Frau traut<. Und ich muss sagen, es stimmt schon irgendwie. Gerade, wenn ich mir so ein paar Mädels aus meinem Freundeskreis angucke oder Frauen generell, fällt mir auf, dass Frauen ein Problem haben zu sagen, >Hier bin ich, das mache ich und dafür möchte ich anständig bezahlt werden«« (E: 6). 
Die interviewten DJs erklären den Erfolg von Musikern nicht vorwiegend durch eine etwaige Bevorzugung eines Geschlechts oder durch entsprechende Netzwerke. Sie verstehen das Auflegen als ein Handwerk, mit dem man durch gute Leistung auch erfolgreich sein kann. Beziehungen spielen eine Rolle, sind aber nicht an das Geschlecht gebunden. Dennoch wird eines der Hauptargumente für die Unterrepräsentanz weiblicher DJs mit der Sozialisation von Mädchen in Zusammenhang gebracht.

Auf die Frage, weshalb es wenige auflegende Frauen im Technobereich gebe, wird meist geantwortet, dass Frauen vermutlich keinen Drang zum »Rumtüfteln« verspürten und die Technik somit als Hindernis sähen. Dieses Desinteresse sei wohl in einer »Technikangst« bzw. »Technikscheu« begründet, welche sich schon im frühen Alter bemerkbar mache: »Vielleicht haben manche Mädchen Schwellenangst [...]. Vielleicht ist es tatsächlich noch so, gerade bei ganz jungen Mädchen. Dass sie denken, >Oh, Technik«« (E: 8). Aber nicht nur beim Auflegen, sondern vor allem beim Produzieren von elektronischer Musik ist die Technik anscheinend ein Hindernis:

»Wobei es mehr Frauen beim Auflegen als bei der Produktion gibt. Es gibt noch nicht viele Frauen, die produzieren. [...] Ich schätze, das liegt am technischen Hindernis. Ich kann mir vorstellen, dass die Technik Frauen abschreckt, wenn sie nicht wirklich daran interessiert sind « (F: 6f.).

Teils scheint diese Technik-Scheu aber auch von Frauen bewusst gewollt zu sein:

»Aber ich muss auch sagen, dass es mir so vorkommt, als würden Frauen manchmal auch kein Technikverständnis haben wollen oder sich eben nicht trauen, weil sie denken, es wäre so schwierig« (E: 6).

»Das hat einmal damit zu tun, dass viele durch Erziehung und fehlende weibliche Vorbilder [...] leider technikscheu sind« (A: 5f.).

Hier kommt neben der Technikphobie der Aspekt einer geschlechtsspezifischen Sozialisation zum Tragen, was sich wohl gegenseitig bedingt.

Ferner wurden die DJs nach generellen Erfahrungen befragt, bei denen sie das Gefühl hatten, als Frau anders behandelt zu werden. So wurde eine von Männern entgegengebrachte Skepsis angemerkt:

»Es gibt manchmal Kommentare von Kollegen, die sagen, >die wird ja nur gebucht, weil sie einen süßen Arsch hat<, oder >weil sie so hübsch ist und deshalb hat sie es viel leichter . Die Männer neigen manchmal schon dazu, es ein bisschen zu reduzieren. Man hat manchmal das Gefühl, dass die Männer sich bedroht fühlen durch die Frauen, dass an ihrem Ego gekratzt wird« (F: 4). 
Eine andere erzählt, dass sie manchmal den Eindruck habe, als würde zwischen Mann und Frau unterschieden werden:

»Zum Beispiel spiele ich ja einmal im Monat im `U60311 und da finde ich es manchmal komisch, wenn Männer, die da öfter spielen, meinen, sie müssten mir noch was erklären oder mich eines besseren belehren. Da frage ich mich schon, ob das auch so wäre, wenn statt mir ein Mann reingekommen wäre« (B: 6).

Bemerkenswert ist, dass es für die Interviewpartnerinnen selbstverständlich ist, dass Frauen hinter den Plattentellern stehen - sie selbst sind eher überrascht, wenn manche Männer das für befremdlich halten:

»Männer, für die es nicht so normal ist, dass eine Frau auflegt, gaffen einen schon an und sind irritiert, [...] und das in der heutigen Zeit! Ich wurde mal gefragt >Wer ist denn dein Freund? und ich habe gefragt >Warum denn? und dann hieß es, >Wessen Plattentaschen ich hier tragen würdeく. [...] Ich denke schon, dass es nicht mehr so besonders ist, dass eine Frau auflegt« (B: 5).

Es kommt klar zum Ausdruck, dass die eigene Wahrnehmung mit der Fremdwahrnehmung nicht übereinstimmt. Für die Frauen ist es nichts Besonderes, dass eine Frau auflegt; auch wenn die Kombination von Frau und Plattenauflegen in der Szene allgemein noch nicht so alläglich zu sein scheint.

\section{Der $\gg$ Exoten-Bonus«}

Dieser Begriff wurde von einer meiner Interviewpartnerinnen erwähnt und bringt die Erfahrungen der anderen Interviewpartnerinnen auf den Punkt. Frauen sind nicht die Norm, sie stellen immer noch Exoten dar. Diese hervorgehobene Stellung bietet aber gleichzeitig einen Bonus - eine Auffassung, die fast alle Interviewpartnerinnen teilen: Zunächst hat man als Frau beim Auflegen einen Vorteil, da man aufällt und somit schneller auf sich aufmerksam macht. Dies kann sich karriereförderlich auswirken, jedoch kommt dieser Vorteil nur durch die Marginalisierung der Frau in der DJSzene zustande. Weibliche DJs werden gerne als »Hingucker « gebucht; dies bedeutet jedoch auch, dass sie aufgrund ihres »Frau-Seins « und nicht unbedingt als Musikerinnen gebucht werden. Dadurch befinden sie sich in einer ambivalenten Situation, denn sie haben keinen Rückhalt von anderen Kolleginnen. Gleichzeitig ist dieses »solistische« Auftreten lukrativ, da sie auffallen und sich dadurch besser am Markt »verkaufen« können: 
»Man will es natürlich nicht wahrhaben, aber es ist schon so, dass man einen >Exoten-Bonus< hat. Wenn du auflegst als Frau, ist es für die Leute immer noch interessanter. Es gibt auch Abende, wo nur Frauen auflegen, das ist für einen Club gute Werbung« (F: 4).

Hierin kommt auch wieder der kommerzielle Aspekt der Frauenrolle zum Vorschein. Auch eine andere Interviewte beschreibt die Reduzierung auf das Erscheinungsbild, aber nicht explizit beschränkt auf das weibliche Geschlecht: »Ich glaube schon, dass man mit Äußerem Pluspunkte macht, Leute finden dich positiv, auch wenn du schlechte Musik gemacht hast « (B: 8). Dieser »Bonus« ist jedoch begrenzt, wie folgende Aussage zeigt:

»Ich glaube, als Frau hat man es am Anfang leicht, weil viele sehen erstmal, >Ah, das ist ' $n$ netter Blickfang und stellen wir sie mal so dahin<. Und wenn es dann um die großen Gagen geht, dann bist du trotzdem so außen vor, manchmal $\ll(\mathrm{D}: 5)$.

Zu dem von Lorenz (2007) beschriebenen »Glass-ceiling «-Phänomen äußerten sich die befragten weiblichen DJs unterschiedlich. Eine der erfolgreicheren meinte:

»Das wird sich mit der Zeit zeigen. Aber man schaut sich Magda an oder Miss Kittin. Insgesamt glaube ich, dass die Szene offen ist und bei den Geschlechtern keine Unterschiede gemacht werden, wie z.B. in den >normalen Berufen. Es gibt eben noch nicht so viele Frauen« (F: 5).

Der Eindruck, dass sich das Gehalt nicht am Geschlecht, sondern an der Popularität orientiert, die anscheinend mit der Erfahrung und der Dauer korreliert, wird nicht von allen bestätigt. Doch gibt es momentan immer noch mehr Männer, die hohe Gagen kassieren. Eine Beobachtung, die Parallelen in der allgemeinen Geschäftswelt hat und Fragen der Emanzipation berührt:

»Wenn man sieht, dass nur 3\% aller Führungskräfte weiblich sind! Das muss man sich mal überlegen, von diesen $100 \%$ sind nur $3 \%$ weiblich! Und so ist es im Grunde in der Musik auch. [...] Es gibt z.B. wenige Label-Chefinnen oder Chefinnen von Booking-Agenturen. [...] Der Grund an sich ist, wie überall auch, dass, wenn man eine bestimmte Machtposition hat in irgendeinem Bereich, dann will man die auch nicht aufgeben. [...] Jetzt drücken zwar Frauen hinterher, aber das dauert noch, bis die dann wirklich da ran kommen. [...] Und dann müssen sie auch noch so sein wie ein Mann. Das ist ja auch noch mal so etwas, eine Leistungssache. Kinder kriegen ist dann auch irgendwie schwierig. Es ist eine Männerwelt, und es hat sich trotz Emanzipation und Frauenbewegung in den letzten 30 Jahren nicht viel in dieser Männerwelt geändert. Es sind zwar Frauen rein gedrängt, aber diese mussten sich dreimal so viel anstrengen und haben dreimal so viel geleistet. Sie mussten sich an 
diese Männerstrukturen anpassen, um das $\mathrm{zu}$ schaffen. Und das ist der Knackpunkt. Diese Strukturen, das sind Männerstrukturen, von Männern gemacht, und das generell aufzuknacken, egal wo das ist, ist sehr schwer. Da arbeiten Frauen dran, schon seit 30 Jahren, und es ist viel passiert! Aber von Gleichberechtigung ist keine Spur. Allein was die Verdienste angeht« (A: 9f.).

»Das ist auch so wie im Bankbusiness, ein bisschen, glaub ich. Ich glaube schon, dass es eine gläserne Decke gibt, manchmal. [...] Ich habe gesehen, dass die Kumpels einfach mehr Geld als ich kriegen. Dann denkste, >Oh, hast du jetzt blöd verhandelt, oder so<. [...] Und man fragt sich, >Warum ist denn jetzt meine Arbeit weniger wert als deren« (D: 5).

\section{Resümee}

Resümiert man die Aussagen der DJs, so kann ein geschlechtliches Ungleichgewicht festgehalten werden, welches für Vermarktungszwecke aber hilfreich sein mag. Als Gründe für das Ungleichgewicht in der Szene wurden unterschiedliche Faktoren genannt; als Hauptargument wurde aber die Sozialisation von Mädchen bezeichnet, welche oft in eine Technikphobie mündet und viele Frauen davon abhält, sich mit dem DJing auseinanderzusetzen.

Die Erfahrungen der interviewten DJs als Mitglieder der Frankfurter Szene waren erstaunlich ähnlich; z.B. wurde von einer skeptischeren Begutachtung von Männern berichtet (im Vergleich zu den männlichen DJ-Kollegen). Den Spruch »Für eine Frau legst du gut auf « haben die meisten von ihnen mehrmals zu hören bekommen.

Netzwerke von Frauen in der Techno-Szene wurden aber tendenziell kritisch betrachtet. So sehen die meisten der Interviewten ein Problem darin, sich lediglich aufgrund des Geschlechts, nicht aber aufgrund eines gemeinsamen Musikgeschmacks zusammenzuschließen. Gleichwohl stellen sie fest, dass es immer mehr Frauen im DJ-Bereich gibt und somit auch Entwicklungen in diesem Bereich möglich sind, da DJ-Frauen wiederum eine Vorbildfunktion für die folgende Generation darstellen.

Der Standort Frankfurt wird als positiv empfunden, um eine DJ-Karriere zu beginnen - jedoch nur bis zu einem bestimmten Punkt. Danach verhelfen nur noch Beziehungen oder der »Umweg « über andere Städte zum weiteren beruflichen Erfolg. Die Kinderfrage wurde als Hauptproblem der DJ-Karriere bezeichnet. Meistens wurde die Vereinbarkeit von DJ-Dasein und Familie nur mit helfendem Partner als realistisch eingestuft, dennoch müsste man mit oder ohne Partner eine »Pause« für Kinder einlegen, die aber für die DJ- 
Karriere aufgrund der Erwartung, immer in der Szene präsent zu sein, schädlich wäre.

Aus Platzgründen bin ich in meinem Beitrag nicht auf ein weiteres interessantes Phänomen eingegangen, möchte es aber an dieser Stelle kurz nennen, da es weitere Forschungsfragen aufwirft. Auffällig war, dass tendenziell eine Abwertung von Frauenbeziehungen geäußert wurde; Frauen wurden oft als »zickig «, »festgefahren« oder »stutenbissig « bezeichnet (vgl. Zimmermann 2008: 64-66). Interessant wäre in diesem Zusammenhang die Frage, weshalb es zu diesen Ressentiments unter Frauen kommt. Ähnliches wurde auch in der Interviewstudie von Stauber (2004: 197) geäußert. Die Gründe hierfür gilt es noch zu erforschen.

\section{Literatur}

Anz, Philipp / Walder, Patrick (Hg.) (1995): Techno. Zürich: Verlag Ricco Bilger.

Braunersreuther, Christine / Maida, Marcus (2000). »Nicht schlecht für eine Frau. Klischees, Rollen, Erwartungen und Erfahrungen von Frauen als Produzentinnen von elektronischer Musik. «In: Testcard. Beiträge zur Popgeschichte, Nr. 8: Gender. Geschlechterverhältnisse im Pop. Mainz: Ventil, S. 56-71.

Coers, Martin M. (Hg.) (2000): Friede, Freude, Eierkuchen. Die Technoszene. München: Beck.

Gardizi, Farid (2006). »From Disco to Disco. Die Kultur des Techno.«In: Jugend in Szenen. Lebenszeichen aus flüchtigen Welten. Hg. v. Doris Lucke. Münster: Westfälisches Dampfboot, S. 177-202.

Gilbert, Jeremy (2006). „More than a Woman. Becoming-Woman on the Disco Floor.« In: Female Consequences. Feminismus, Antirassismus, Popmusik. Hg. v. Rosa Reitsamer und Rupert Weinzierl. Wien: Locker, S.181-193.

Kemper, Christian (Hg.) (2004): Mapping Techno. Jugendliche Mentalitäten der 90er. Frankfurt/M.: Europäischer Verlag der Wissenschaften.

Lorenz, Isabel (Hg.) (2007): Die DJane Bewegung - Weibliche DJs zwischen Aufbruch und Marginalisierung. München, Ravensburg: Grin-Verlag.

Mayring, Philipp (2000). »Qualitative Inhaltsanalyse. «In: Qualitative Forschung. Ein Handbuch. Hg. v. Uwe Flick, Ernst von Kardorff und Ines Steinke. Reinbek bei Hamburg: Rowohlt, S. 468-475.

Stauber, Barbara (Hg.) (2004): Junge Frauen und Männer in Jugendkulturen. Selbstinszenierungen und Handlungspotentiale. Opladen: Leske \& Budrich.

Zimmermann, Corinna Jean (2008). Weibliche DJs in Frankfurt. Eine Interviewstudie zum Geschlechterverhältnis in der elektronischen Musik am Beispiel des Standortes Frankfurt am Main. Frankfurt/M.: Hochschule für Musik und Darstellende Kunst, unveröffentlichte Staatsexamensarbeit. 


\begin{abstract}
Looking at today's DJ scene one might discover a disproportion of sex ratio. The article presents data from an interview survey on six female Techno DJs and their experiences in Frankfurt/Main. The survey focuses on gender related issues such as, why are there more male than female DJs, and is Techno (still) a scene where gender borders do not matter? The survey provides insights in female DJs' perceptions and experiences of today's Techno DJ scene in Frankfurt/Main revealing ambiguous approaches towards gender stereotypes.
\end{abstract}

